M: Die Produktionsdramaturgie bedeutet, dass man gemeinsam mit dem Regisseur ein Konzept erarbeitet. Man könnte sagen, dass der Dramaturg eine Art inhaltlicher Begleiter der Produktion oder auch eine Art Brücke zwischen der Produktion, ins Haus hinein, aber auch zu den Zuschauern ist. Dann gibt es das Arbeitsfeld, das die Spielplangestaltung betrifft, wo man sich natürlich auch wieder konzeptuell überlegt, welche Stücke man inszenieren will, wie man einen Spielplan zusammensetzt, welche Regisseure engagiert werden, und dann natürlich alles, was ein bisschen reingeht in inhaltliche Begleitung der Gesamtveranstaltung. Das heißt, man organisiert Zusatzveranstaltungen wie Lesungen, Diskussionen, man macht auch auswärtig Veranstaltungen, in Schulen zum Beispiel, und spricht mit den Leuten über das, was sie gesehen haben. Das sind die drei großen Arbeitsfelder, wenn man so will.

I: Wie war das jetzt im Fall von Mythos, Propaganda und Katastrophe in NaziDeutschland und dem heutigen Amerika? Was hat Sie überhaupt dazu bewogen, dieses Stück auszusuchen und es jetzt und hier zu zeigen?

M: 'Das war in dem Fall so, dass der Regisseur, Burkhard C. Kosminski, in Dänemark unterwegs war und zufälligerweise dort bei einem Freund dieses Stück auf englisch auf dem Schreibtisch liegen sehen und dann mitgebracht hat. Wir haben das in der Dramaturgie gelesen und fanden das sehr spannend; zum einen natürlich aufgrund der Thematik an sich, zum anderen, aufgrund der Art, wie es reagiert oder sich thematisch befasst mit dem 11. September und Terrorismus aus einer anderen Perspektive. Daher haben wir natürlich sofort gesagt, wir wollen das machen.

I: Ich nehme an, es gab noch keine deutsche Übersetzung zu dem Zeitpunkt. Die deutsche Übersetzung, die sie dann verwendet haben, stammt von John und Peter von Düffel; wie ist das zustande gekommen?

M: Das war jetzt in dem Fall auch wieder ein Ausnahmefall. Wie gesagt, Burkhard Kosminski hat dieses Stück mitgebracht und es dann dem Rowohlt-Verlag angeboten. John von Düffel ist ja auch beim Rowohlt-Verlag und arbeitet sehr eng mit denen zusammen, so dass es über den Verlag gelaufen ist; die haben dann den Übersetzer bestimmt.

I: Das Stück ist, soweit ich weiß, in Amerika noch nicht aufgeführt worden, sondern nur in England und Australien. Glauben Sie, dass es noch aufgeführt werden wird? Das Stück ist ja nicht ganz ohne Sprengkraft.

Stephen Sewell: Mythos, Propaganda und Katastrophe in NaziDeutschland und dem heutigen Amerika (Myth, Propaganda and Disaster in Nazi Germany and Contemporary America, 2003)

\title{
Hannah Vater
}

Interview mit Sybille Meier, Dramaturgin am Schauspielhaus Düsseldorf (21.06.05)

M: Schwierige Frage. Ich weiß nicht, ob es da eine Zensur gibt, was man vielleicht gerne unterstellen möchte, oder prinzipiell würde ich sagen, wie Amerika hier auch oft gesehen wird. Ich bin mir nicht sicher, ob das damit etwas zu tun hat, oder ob es einfach schlicht und ergreifend daran liegt, dass sich bislang kein amerikanischer Regisseur oder kein Theater dafür interessiert hat.

I: Aber jetzt hier in Düsseldorf gab es keine Bedenken, das Stück aufzuführen? 
M: Nein. Die einzigen Bedenken, die es gab, waren ganz praktischer Natur, und zwar was den Titel betrifft: Mythos, Propaganda und Katastrophe in NaziDeutschland und dem heutigen Amerika. Das ist natürlich ein relativ ungewöhnlicher Titel, weil er ja wirklich ein wissenschaftlicher Titel ist und eigentlich kein dramatischer Stücktitel. Da gab es in der Tat Diskussionen, ob man noch mal mit dem Autor sprechen sollte, aber der Regisseur und ich waren uns darüber einig, dass wir gerade diesen Titel sehr gut finden. Es gab aber jetzt nicht die Frage, ob das politisch so brisant oder so provokativ sei, dass man dieses Stïck nicht hier aufführen könnte.

I: Sind Sie oder waren Sie mit Stephen Sewell in Kontakt oder haben Sie einfach nur den Text bekommen und dann selber weiter bearbeitet?

M: Ja, wir haben nur den Text bekommen und nicht mit ihm zusammengearbeitet. Man muss dazu sagen, es gibt einen sehr großen Unterschied in der englischsprachigen Theatertradition und in der deutschsprachigen Theatertradition, nämlich das seit den 80er Jahren so genannte Regietheater. Ein weiter Begriff, dennoch will ich kurz was dazu sagen. Es ist einfach so, dass es im englischsprachigen Raum nicht üblich ist, den Text als Partitur oder Material zu begreifen, wo man kürzen, streichen, hinzufügen, oder kollagieren darf - das gibt in Deutschland manchmal auch Probleme, je nachdem wie die Erben reagieren, aber selten. Diese Tradition gibt es im englischsprachigen Raum einfach gar nicht, das heißt, dort ist es komplett aufgeführt worden. Wie sie sicherlich bemerkt haben, haben wir zum Beispiel über vierzig Prozent des Textes eingestrichen.

I: Ja, mir ist aufgefallen, dass zum einen sehr viel gestrichen wurde und zum anderen, wenn man sich etwa das Programmheft ansieht, befindet sich der Schwerpunkt sehr auf der politischen Seite, insbesondere auf dem Demokratieverständnis. In den Szenen, die Sie nicht benutzt haben, geht es dagegen ein bisschen mehr in die Psyche. Woher kommt das eigentlich beim Menschen, dieses Bedürfnis; wo kommt diese Angst her und die Reaktion auf die Angst? Da mussten Sie wahrscheinlich schon eine Entscheidung treffen.

M: Ja, es ist einfach so, dass wir fanden, als wir das Stück gelesen haben, es erklärt sehr viel und spricht auch sehr viel aus und bietet sozusagen auf zwei Strängen Erklärungsmodelle an: nämlich zum einen, wie Sie schon gesagt haben, möglicherweise begründet in der Psyche einer Figur, und das andere ist einfach mehr im Vagen gelassen. Dieses Sich-selbst-erklären einer Figur, das hat uns daran nicht so gefallen, das haben wir dann auch tatsächlich weg gestrichen. Also beispielsweise die Szene beim Psychiater, die, wie ich finde, auch eine Doppelung ist. Das muss man nicht alles noch mal aussprechen, um zu begreifen, was eigentlich mit dieser Frau los ist, sowohl psychologisch als auch in dem Kontext dieser Welt, in der sie sich da bewegt.
I: Wenn man betrachtet, wie die Machtausübung in der amerikanischen Demokratie funktioniert, kann man das auf deutsche Verhältnisse übertragen? Oder muss man die Verhältnisse in Amerika kennen, um das Stück zu verstehen?

M. Ich denke, natürlich muss man sie kennen, man muss natürlich auch diese zweite Ebene der Handlung, was diesen Campus betrifft verstehen. Ich glaube schon, dass man eine Vorstellung davon haben sollte, was z.B. ,sexual harassment' bedeutet, was wir ja hier in der Form oder immer in diesen extremen Auswirkungen in Deutschland nicht haben. Was die politische Situation betrifft, glaube ich - man kann es wahrscheinlich gar nicht unterscheiden - aber die Amerika-rezeption ist seit dem zweiten Weltkrieg so groß, dass man davon ausgehen kann, dass jeder diese Verhältnisse einigermaßen kennt. Zur Frage der Übertragung: Was uns eben an dem Stück sehr interessiert hat - und da schlägt es ja dann auch letzten Endes den Bogen zu unserer Kultur, zu unserer Literatur - natürlich einmal, was Kafka betrifft, und zum anderen die Frage, wie geht man mit Paranoia um. Das ist, glaube ich, das ganz große Thema darin, dass zum Beispiel die Medien in Amerika vielleicht noch in einer stärkeren oder anderen Weise das ihrige tun, nur tendenziell passiert bei uns ja genau das gleiche, sprich, man kriegt die scheinbare Realität vermittelt durch Fernsehen, Zeitung etc. und macht sich dann ein Bild, z.B. dieser Mythos vom Schläfer, der da irgendwo hier durch unsere Städte geistert und dann plötzlich eines Tages erwacht und dann irgendwas tut. Das ist das eigentlich spannende Thema und ich glaube, dass das allgemeingültig für die westliche Welt ist und überhaupt nicht amerikaspezifisch.

I: Denken Sie, dass man mit so einem Stück die Leute zum Nachdenken bewegt oder ist Theater mittlerweile dazu nicht mehr in der Lage? In London liefen vor kurzer Zeit Stücke - eines mit dem Titel Guantanamo - die in eine ähnlich Richtung gingen, also sehr politisch sind, die, wenn man da an Brecht denkt, nicht mehr unterhalten wollen, sondern die Leute wirklich zum Nachdenken anregen.

M: Ja, davon gehen wir mal aus, wenn wir ein Stück machen (lacht), dass man natürlich so ein Stück auf den Spielplan setzt, um nachzudenken. Ich möchte aber dazu sagen, Theater ist ja nicht nur ein Ort der Reflexion, sondern ist natürlich auch immer ein sinnliches Erlebnis, und das unterscheidet es von irgendwelchen wissenschaftlichen Diskussionen oder sonstigem. Und sich in die Lage dieses Mannes zu versetzen - wobei ja durchaus nicht entschieden sein will, ob es nich tatsächlich auch so sein könnte oder eines Tages passiert sein könnte - das heißt einfach noch mal über einen anderen Weg dieses Nachdenken, wenn sie so wollen, gleichzeitig über ein Mit-Erleben einfach in Gang zu setzen.

I: Jetzt habe ich noch einige Punkte, die mir zur Inszenierung selber aufgefallen sind: Und zwar einmal, dass spezifische Orte in der Inszenierung gar keine Rolle mehr spielen, z.B. bei dem Abendessen, wo die Schauspieler einfach nur nebeneinander stehen und nach vorne gucken. Was kann man zu dem Raum oder Ort auf der Bühne sagen? 
M: Es ist so, dass die Intention ist, nicht illusionistisch zu arbeiten, weil das ohnehin, glaube ich, völlig theaterfremd ist. Das kann man im Film machen, aber nicht im Theater. Insofern versucht man das, was diesen Ort wesentlich bestimmt, auf die Bühne zu bringen. Das war der Versuch, dass diese Menschen, die Schauspieler, quasi während des ganzen Abends da sind und sich in einem Raum befinden. Sie haben's gesehen: dort waren diese Scheinwerfer, sie stehen also die ganze Zeit im Licht, sind beteiligt am Geschehen, und werden zugleich von diesen Medien, also sehr dominanten Monitoren eingerahmt. Es sollte die Situation umgesetzt werden, beim Zuschauer evoziert werden, dass man also einerseits permanent in der Beobachtung steht, quasi wie so eine Versuchsanordnung, und gleichzeitig auch immer mit beobachtet. In dem Fall hat man die Situation, dass der Schauspieler auch da sitzt und das Publikum beobachtet, was normalerweise nicht der Fall ist, weil er ja abgeht, wenn er nicht mehr mitspielt. Das ist eigentlich die Idee des Raumes gewesen.

I: Ich habe zwei Kritiken in deutschen Zeitungen gelesen. Dort stand z.B. über die Videoscreens, dass sie den surrealen Charakter verstärken sollen. Ich hätte jetzt eher vermutet, das das heißen soll, das die Medien einfach ständig und immer präsent sind.

M: Ja, das würde ich auch so sehen.

I: Etwas, das mir noch aufgefallen ist, war, dass die Studentin, Margurite, deutlich lasziver wirkte als im Text dargestellt.

M: Ja, da war einfach die Idee, dass das merkwürdige an dieser Studentin ist, dass sie einerseits sehr intellektuell, sehr politisch engagiert ist, andererseits aber diese erotische Dimension im Stück ja durchaus vorhanden ist. Ich meine, der Autor spielt ja auch in den Szenen damit, dass man zunächst gar nicht ganz genau weiß, was die beiden eigentlich voneinander wollen - ist da in der Tat ein sexuelles oder erotisches Interesse vorhanden oder nicht? - und dann kommt es irgendwann $\mathrm{zu}$ diesem Knall. Dieses Spiel wollten wir einfach auch weiter drin lassen, um das nicht eindeutig so zu zeigen, dass man denkt, es sei ohnehin nur eine rein intellektuelle Beziehung.

I: Die interessanteste Figur ist ja eigentlich der „Mann", dessen Identität nie geklärt wird - was ja auch gemäß Kafka nicht Sinn der Sache ist. In der Kritik wurde bemängelt, der Mann würde störende Mätzchen machen, dass er mit Piepsstimme oder mit Hitlerstimme spricht, dass er eine Elvismaske trägt usw. Wie sollte der Mann dadurch charakterisiert werden, wozu dienten diese Einlagen?

M: Das ist aus Probenimprovisationen entstanden. Es ist so, dass man lange bevor man tatsächlich auf die Bühne rausgeht, in der Probenarbeit über die Figuren spricht. Das war eben der Punkt, dass man diesen Mann nicht genau als CIA-Typen zeichnen wollte, also nur im schwarzen Anzug, der sowieso von Anfang an klar gewesen wäre. Dann gibt es eine Sache, die natürlich filmisch immer sehr einfach zu lösen ist, nämlich dieses Spiel mit Realität und Fiktion, wo man sofort an Filme denkt wie A Beautiful Mind. Will sagen, auf der Bühne muss man ja anders umsetzen, da muss man schon mit anderen Mitteln arbeiten, weil man nicht einfach jemanden verschwinden lassen kann oder schneiden. Und das war die Idee, diesen Mann gerade nicht als Figur zu zeigen, die eindeutig irgendwas ist, sondern man weiß es nicht genau, man kann ihn nicht einordnen. Er wollte eben gar keine Figur sein, daher diese verschiedenen, Mätzchen, wie es der Kritiker nennt oder die Arten irgendetwas zu sprechen, um eben nicht in einen Charakter überzugehen.

I: Bei der Darstellung des Talbot fällt auf, dass er in den letzten Szenen sehr mechanisch auf die Fragen des Mannes antwortet, so als würde er es vom Band abspulen. Das hat mich ein bisschen gewundert, denn er ist ja in einer sehr bedrängten Situation.

M: Sie meinen die Szene, als er da auf dem Stuhl sitzt. Das war eigentlich nicht so, dass er mechanisch antwortet, sondern das war, dass er quasi in sich gefallen ist, so eine Art wie, ja, man ist in sich versunken und nimmt sein Außen gar nicht mehr wahr, ein bisschen, wie wenn man gerade erwacht ist, zum Beispiel, und noch nicht genau den Wachzustand erreicht hat. So sollte es eigentlich sein.

I: Auch ganz am Ende, als er ja im Prinzip die Oberhand gewinnt - obwohl er dann erschossen wird - und sich intellektuell eigentlich durchsetzt, da spricht er immer noch auf diese Weise. Der Mann dagegen wurde gegen Ende sichtlich nervös.

M: Bei uns ist das Ende ja offen geblieben. Es wird nicht eindeutig - wenn Sie jetzt sagen, dass er erschossen wird - bei uns ist eigentlich der Gedanke, es ist gar nicht klar, ob er tatsächlich erschossen wird.

I: Es gab keinen Knall.

M: Genau. Also, es ist keine Illusion eines Schusses, sondern es bleibt offen, ob das nun in der Tat ein einziger Alptraum, ein einziges Hirngespinst von ihm war oder ob es tatsächlich stattgefunden hat.

I: Ganz am Schluss ist ja eigentlich der Clou, dass Eve den Zündschlüssel von Talbots Auto umdreht und dieses explodiert - eine Ahnung, die Talbot selbst vorher nebenbei geäußert hat und die eher paranoid wirkte. Daraufhin wird eine Nachrichtendurchsage eingespielt, sie sei eine Selbstmordattentäterin gewesen und eine „muslimische Studentin" als ihre Mittäterin verhaftet worden. Leider hat man diese Durchsage akustisch sehr schlecht verstanden.

M: Da gab es eine lange Diskussion, da rennen Sie jetzt bei mir offene Türen ein, weil ich das auch gedacht habe. Das Problem der Technik war, wenn man da jetzt quasi noch mal eins draufsetzt, ist das schwierig, weil es dann immer auch ein bisschen ins Plakative geht und da wollten die das nicht absetzen. Wie gesagt, das sind manchmal Entscheidungen, natürlich möchte man nicht, dass man es nicht versteht, aber es gab eben Leute, die haben es verstanden und gesagt, damit haben sie überhaupt kein Problem. Ich persönlich hatte damit auch ein Problem und 
deswegen verstehe ich jetzt, was Sie sagen. Es gibt da ganz unterschiedliche Reaktionen, das war dann einfach eine Entscheidung, dass das so zu Ende geht.

I: Wurden am Düsseldorfer Schauspielhaus eigentlich schon andere australische Dramen der Gegenwart aufgeführt?

M: Also meines Wissens nicht. Es gibt, glaube ich, keine wirkliche Tradition der australischen Gegenwartsdramatik, das ist eigentlich eher unbekannt. Man hat ja oft einen Bezug zu englischen Dramen - also, kennen Sie ja wahrscheinlich, Sarah Kane usw. - die seit einigen Jahren auch in Deutschland regelmäßig auf dem Spielplan auftauchen, aber australische Gegenwartsdramatik ist weitgehend unbekannt. Das war jetzt mit das erste, was uns da begegnet ist. Und das auch noch auf einem dänischen Schreibtisch. 